\title{
(RE)EXAMINANDO HORIZONTES EN LOS ESTUDIOS FEMINISTAS DE TRADUCCIÓN: ¿HACIA UNA TERCERA OLA?
}

\author{
Olga Castro
}

Universidade de Vigo

\begin{abstract}
Feminisms are one of those framework theories that have contributed powerfully to all areas of society, including Translation Studies. The most evident outcome of this interplay is the emergence, in the 1980s, of a Feminist Translation school in Canada, which placed gender in the spotlight. Despite criticism and subsequent redefinitions of the notion of feminist translation, the Canadian school is still generally regarded as the paradigm of interaction between feminisms and translation. The aim of this article is two-fold: firstly, to advance new approaches to the practice of translation and paratranslation from a feminist perspective (within the context of a third wave of feminist translation). Secondly, to open new debates by means of (re)examining topics of mutual interest for both Translation Studies and Feminisms on a conceptual, historical and critical plane, so that subsequent studies can be fostered.
\end{abstract}

\section{Resumen}

Los feminismos son una de esas teorías marco cuyas contribuciones son perceptibles en todos los ámbitos de la sociedad, incluidos los estudios de traducción. La materialización más evidente de esta interacción es el surgimiento, en los 80, de una corriente de traducción feminista en Canadá, capaz de colocar el género en el centro del debate sobre traducción. En la actualidad, y pese a las críticas y posteriores redefiniciones del concepto de traducción feminista, la propuesta canadiense sigue concibiéndose por lo general como paradigma de interacción entre feminismos y traducción. En este artículo propongo nuevas aproximaciones a la práctica de traducir y paratraducir desde los feminismos, dentro de una tercera ola de traducción feminista. Además, pretendo abrir el debate (re)examinando áreas de interés mutuo para los estudios de traducción y los feminismos en el plano conceptual, historiográfico y crítico, con el propósito de que sugieran nuevas líneas de investigación futura. 
Keywords: Feminist translation. Feminist paratranslation. Ideology. $3^{\text {rd }}$ wave feminism. Gender and translation.

Palabras clave: Traducción feminista. Paratraducción feminista. Ideología. Tercera ola feminista. Género y traducción. 
En el contexto de las múltiples teorías post- (postcolonialismo, postmodernismo, postestructuralismo) de los años 70 , y de un renovado interés por los estudios culturales, se produce un encuentro entre los feminismos y los estudios de traducción (ET) que enriquecerá a ambas disciplinas. Una de las materializaciones de esta intersección se encuentra en el nacimiento de la escuela de la traducción feminista canadiense. Su contribución a los ET fue (y es) tal que, pese a las críticas y posteriores redefiniciones de la noción de traducción feminista, en la actualidad todavía es frecuente que desde las corrientes dominantes de la traducción se conciba la propuesta canadiense como paradigma universal de la traducción feminista y, por extensión, como paradigma de la interacción entre los feminismos y la traducción.

Sin embargo, en un momento como el actual, en el que los feminismos se hallan en un proceso de debate interno tras la constatación de que el feminismo de la segunda ola (el que había inspirado a la escuela canadiense) no sirve como marco en el que desarrollar las nuevas propuestas, sostengo que se dan las circunstancias propicias para ampliar los ámbitos de estudio que puedan surgir de la interacción entre ambas disciplinas ${ }^{1}$. Propongo en primer lugar aunar y reexaminar desde una perspectiva crítica algunas de las áreas de interés mutuo entre feminismos y traducción que, aun habiendo sido estudiadas, carecen de sistematización al haber quedado ensombrecidas por el carácter central (dentro de los márgenes) de la propuesta canadiense. Pretendo en este punto ir más allá del nivel fundamentalmente práctico de éstos para abarcar también el nivel conceptual, historiográfico, crítico o profesional, lo que ofrecerá una comprensión más holística de los puntos de intersección entre feminismos y traducción con el afán de contribuir a impulsar estos renovados horizontes de investigación. En segundo lugar, propongo reexaminar el nivel práctico abogando por nuevas aproximaciones feministas al acto de mediación ideológica intercultural que es la traducción y la paratraducción (Garrido 2005), dentro del marco que nos ofrece la lingüística feminista de la tercera ola (Mills 2003 y 2008).

\section{El encuentro entre feminismos y traducción}

Una de las muchas contribuciones de los feminismos al conocimiento se halla en la revisión crítica a la que han sometido las diferentes disciplinas científicas

1. Utilizo el término disciplina por razones prácticas, aunque con ciertas reservas, ya que las múltiples interrelaciones que tanto los feminismos como los estudios de traducción con otras áreas de conocimiento harían más preciso denominarlas interdisciplinas o transdisciplinas. 
y humanísticas, para cuestionar su carácter supuestamente neutro y objetivo y desvelar que respondían (en diferente medida) a criterios patriarcales. Sin embargo, la revisión de la traducción por parte de los feminismos contó desde el principio con una particularidad añadida, puesto que la propia disciplina a revisar se encontraba ya en pleno proceso de debate interno para adaptarse a las nuevas concepciones filosóficas de la época. Así, en ese momento la traductología asistía al nacimiento de nuevos enfoques que centraban el estudio de la traducción no en el producto en sí, sino en el proceso de traducción, en tanto en cuanto de él depende el producto:

The purpose of translation theory is to reach an understanding of the processes undertaken in the act of translation and, not, as so commonly misunderstood, to provide a set of norms for effecting the 'perfect' translation. (Bassnett 1991: 37)

Los estudios descriptivos comenzaron a cuestionar las teorías que se centraban eminentemente en enumerar técnicas con las que poder realizar un relevo lingüístico pasando de las estructuras superficiales de un texto a las de otro con la mínima interferencia posible para guardar fidelidad a la intención del autor/a y al texto original. En su lugar, los nuevos enfoques consideraron "the orientation towards cultural rather than linguistic transfer" (Snell-Hornby 1990: 82), conduciendo así a un "cultural turn" o giro cultural de la traducción, que supone la integración de la dimensión cultural "making language work as a parallel system to culture instead of as an external referential entity" (Nouss 2000: 1351).

Estas nuevas aproximaciones comenzaron también a interrogar el papel hasta entonces neutro, objetivo e invisible de la persona que traduce, y a reivindicar su papel activo, pues el primer paso al traducir (o reescribir, para decirlo con Lefevere y Bassnett 1990: 10) consiste en leer un texto original escrito por una figura autorial que debe ser consciente de la existencia de varias (aunque no infinitas) lecturas e interpretaciones posibles a partir de sus textualidades. Se presenta, pues, como irreal la posibilidad de producir un texto equivalente y fiel al original o a la autoría. Para estos nuevos enfoques no se traducen palabras, sino significados; y éstos no se hallan ni en el texto original ni en la intención autorial, sino que son fruto de negociaciones dentro del sistema social en el que el texto se produce y consume. Por ello, a lo único que se puede ser fiel es a la interpretación que cada traductor/a realice (del original o de la autoría) a través de su lectura.

La ideología se considera un concepto relevante en el acto de traducir, entendida no como desviación de la objetividad, sino como conjunto sistemático de valores y creencias compartidas por una comunidad dada, que conforman 
las interpretaciones y representaciones del mundo de cada persona y también de quien traduce. De hecho, contemplar la ideología como ente ajeno a quien traduce dejaría a este agente mediador y al propio proceso fuera del intercambio cultural. La objetividad y neutralidad en traducción son falacias interesadas, y de este modo, el giro cultural podría igualmente denominarse giro ideológico, por lo que desde nuevas corrientes como la Escuela de la Manipulación o la Teoría de los Polisistemas se defiende que "ideology rather than linguistics or aesthetics crucially determines the operational choices of translators" (Cronin 2000: 695).

En definitiva, cuando se produce el acercamiento de los feminismos a la traducción, ésta había ya superado (al menos en su discurso teórico) el debate sobre la fidelidad, equivalencia y objetividad, y se estaba preguntando por cuestiones que obligaban a reflexionar sobre problemas culturales e ideológicos. El análisis de la realidad desde el prisma de la cultura y de la ideología llevaba tiempo ya en la agenda de los feminismos, que de esta forma conciben su relación con la traducción como mutuamente enriquecedora. De un lado, los feminismos se alimentaron de nuevas ópticas con el debate que estaba teniendo lugar en los ET. Del otro, los ET supieron ver cómo la conexión con esta disciplina contribuía a consolidar sus propuestas, pero además supieron enriquecerse con la perspectiva de género aplicada a afirmaciones como "all translation implies a degree of manipulation of the source text for a certain purpose" (Hermans 1985: 11). Así, los feminismos constataron que el hecho de no suscribir de forma consciente una ideología particular en traducción implica adherirse de forma inconsciente a la ideología dominante (patriarcal), aquélla que poseen todas las sociedades, y que es dominante tanto en sentido numérico como porque apoya los intereses de la clase dominante, lo que le obliga a disfrazarse y operar al nivel del inconsciente (Althusser 1975). Por ese motivo, se presenta ante el traductor o traductora como "normal", "natural" e incontestable, logrando así su propósito de dominación simbólica (Bourdieu 1998) que convierte a las y los traductores "inconscientes" en ingenuos vehículos de transmisión y legitimación del discurso dominante, con el prejuicio añadido de que la ideología es más efectiva cuando no se manifiesta explícitamente como tal.

\subsection{La traducción feminista canadiense}

En paralelo al desarrollo de este nuevo marco teórico-metodológico, surge una nueva corriente ubicada en el diálogo cultural anglo-francés de Canadá que identifica la traducción como la conjugación entre una teoría practicante y una práctica teorizante desde la que examinar cuestiones culturales e 
ideológicas. La traducción feminista canadiense (cf. Godard 1990; LotbinièreHarwood 1991; von Flotow 1991 y 1997; Simon 1996; Vidal 1998: 101-120) consiste en una corriente de trabajo y pensamiento que defiende la incorporación de la ideología feminista a la traducción por la necesidad de articular nuevas vías de expresión para desmantelar la carga patriarcal del lenguaje y de la sociedad. El traductor y traductoras feministas canadienses (Howard Scott, Barbara Godard, Marlene Wildeman, Susanne de Lotbinière-Harwood o Luise von Flotow) traducían a inglés textos literarios vanguardistas de autoras francófonas de Québec, caracterizados por atacar desde la con(s)ciencia las convenciones misóginas del lenguaje patriarcal y por construir una cultura literaria feminista paralela, todo ello reflejando una fuerte influencia de las teorías posmodernas del lenguaje. A partir de estos textos las traductoras canadienses conciben la traducción como una continuación del proceso de creación y circulación de significados dentro de una red contingente de discursos. Dadas las características inherentes del francés (que con su género gramatical explicita constantemente el sexo de las o los referentes) y del inglés (con muchos neutros y epicenos), las traductoras feministas innovaron para encontrar nuevas fórmulas de expresión que no borrasen las marcas de género del original (así, por ejemplo, autoras se convierte en authers en lugar del genérico authors). Las estrategias que utilizaron fueron sistematizadas posteriormente como supplementing, prefacing, footnoting y hijacking the text ${ }^{2}$ (von Flotow 1991: 74-84), y con ellas reivindicaban la visibilidad de las traductoras: "womanhandling the text in translation means replacing the modest, self-effacing translator" (Godard 1990: 93). Entendían la traducción como una "rewriting in the feminine" convertida en "political activity aimed at making language speak for women" (Lotbinière-Harwood 1991).

Fuera de Canadá estas estrategias las utiliza Suzanne Jill Levine (1983) para traducir a inglés textos posmodernos "oppressively male, narcissistic, misogynistic and manipulative" del autor latinoamericano Cabrera Infante. Su opción es convertirse en una "subversive scribe" que reescribe el texto de forma subversiva siendo fielmente infiel.

\subsection{Críticas y redefiniciones}

La aportación de esta escuela a los ET fue notable, ya que insistió en la necesidad de reflexionar de forma consciente y crítica sobre los elementos presentes

2. Massardier-Kenney (1997) demuestra cómo las estrategias canadienses no son invenciones, sino readaptaciones de otras estrategias utilizadas (incuestionadamente) durante siglos. 
en el texto a traducir antes de reescribirlo, impugnó el paradigma tradicional basado en la réplica absoluta problematizando la relación TO/TT, y abogó por una visibilidad traductora. Sin embargo, sus estrategias han sido objeto de diversas críticas ${ }^{3}$ que las acusaban de caer en el "infamous double standard" de las teorías tradicionales de traducción (Arrojo 1994: 149) y de aplicar una ética hipócrita y contradictoria (Arrojo 1995). En resumen, para esta autora:

they are perfectly legitimate within the political context they are so bravely fighting to construct $[. .$.$] However, they are not absolutely more 'noble' or$ more justifiable than the patriarchal translations and notions they are trying to deconstruct. (1994: 159)

Además, proclamas como que su objetivo consiste en "making language speak for women" en todas las circunstancias acaban relacionando la traducción feminista con una actitud esencialista basada en una cultura femenina distintiva que borra las diferencias entre las propias mujeres, y con una definición universal y estable de las mujeres como grupo oprimido.

Surgieron entonces otras concepciones de traducción feminista para superar el sesgo esencialista anterior. En este sentido hay que entender las propuestas que, partiendo de la diversidad de mujeres y experiencias, exploran diversas formas de "traducir en femenino". Maier (en Godayol 1998: 161) considera su posición como "woman-identified translator" o "gender-conscious translator", porque sus traducciones no tienen por qué identificarse como mujeres, sino con mujeres. También Díaz-Diocaretz reflexiona sobre su traducción feminista de Adrienne Rich (1985). Massardier-Kenney (1997), por su parte, propone directamente una "redefinition of feminist translation practice" que contemple estrategias basadas en la autoría y en la traductora.

A pesar de estas críticas y redefiniciones, en la actualidad todavía es frecuente que desde las corrientes dominantes de los ET se conciba la traducción feminista canadiense como el paradigma de interacción entre feminismos y traducción, lo que visto desde una perspectiva (auto)crítica resulta contraproducente en tanto que coarta el desarrollo productivo de otras áreas de investigación.

\section{2. (Re)examinando horizontes: viejas y nuevas interacciones}

Aun reconociendo que las propuestas canadienses contribuyeron de manera significativa a los ET abriendo nuevos y necesarios interrogantes sobre el acto ideológico de traducir que colocaban el género en el centro del debate,

3. Otras críticas patriarcales simplemente las ridiculizan sin aportar argumentos convincentes. 
y reconociendo también que resultaron muy productivas en sus contextos específicos, defiendo que para potenciar el debate y las interrelaciones de los ET con otras disciplinas, se deben ampliar los ámbitos de estudio que puedan surgir de la interacción de éstos con los feminismos. Una buena forma de hacerlo, a mi juicio, es comenzar por recopilar en áreas temáticas algunos de los trabajos realizados en el pasado sobre otras confluencias entre feminismos y traducción para posibilitar una comprensión más íntegra de la interacción, e invitar a retomar con aires renovados estas áreas de estudios que incluyen, además de un revisado plano práctico, el plano conceptual, historiográfico y crítico.

\subsection{Plano conceptual}

Es importante examinar desde una perspectiva feminista los discursos teóricos sobre traducción, no únicamente por justicia social, sino por el potencial de estos discursos en la formación de profesionales, a través de su lectura (sumisa o crítica). Rechazar la teoría implica negar la necesidad de una crítica de la realidad, y por ello el objetivo del marco teórico es ayudar a reflexionar sobre cómo mejorar la práctica (Vidal 1998: 120), a entender la dimensión de la traducción, y a comprender los límites y libertades que conlleva su práctica.

\subsubsection{Metaforización de la traducción}

A lo largo de la historia se ha recurrido con frecuencia a metáforas para explicar el acto de traducir, pues como apunta D'hulst (1992) existe algo sobre la experiencia de traducir que demanda un lenguaje metafórico. Lo que revela Chamberlain en su influyente ensayo "The Metaphorics of Translation" (1992) es que buena parte de los discursos teóricos sobre traducción se han basado en concepciones misóginas sobre los roles de género (legitimándolos). Una de estas frecuentes y conocidas metáforas es la de "Les belles infidèles", expresión acuñada por Gilles Ménage a principios del siglo XVII en Francia para describir que las traducciones, al igual que las mujeres, si son bonitas (belles), serán infieles (infidèles).

El concepto de fidelidad y la preocupación por el origen/originalidad del texto de partida está presente en muchas otras metáforas que se reflejan en el lenguaje cotidiano sobre traducción: paternidad del texto, penetración del texto de partida, traducción fiel, traición de la lengua, etc. Sumada a la noción de propiedad (la mujer es propiedad del hombre igual que el texto es propiedad de su autor), se han creado metáforas para sostener que, en el matrimonio como en la traducción, únicamente una promesa de fidelidad podía garantizar 
la legitimidad, y sin ella los traductores puedan engendrar bastardos textuales (Schleiermacher, apud Godayol 2000: 44).

Otras metáforas recurren a la violencia para explicar el proceso de traducción. Drant, en el prólogo a su traducción de Horacio, justifica la 'violación' del original al compararla con la purificación que lleva a cabo un marido (el traductor) para preparar a una mujer cautiva (el texto de Horacio) y penetrarla, secuestrarla, hacerla suya, convertirla en su esposa:

First, I have now done as the people of God were commanded to do with their captive women that were handsome and beautiful: I have shaved off his hair and pared off his nails, that is, I have wiped away all his vanity and superfluity of matter. (apud Chamberlain 1992: 61)

Las metáforas sexistas no se encuentran únicamente en la época clásica. El modelo hermenéutico de Steiner presenta igualmente un proceso traductor en cuatro fases, "as a hermeneutic of trust, of penetration, of embodiement and of restitution" (Steiner 1992: 319), con un lenguaje erótico y un modelo sexuado donde metafóricamente el hombre es el traductor y la mujer, la traducción. La clásica retórica sexuada la utiliza también Derrida, cuando propone un contrato de traducción (como el del matrimonio) por el que ésta se casa con el original para comple(men)tarse en otro nuevo texto que garantice la supervivencia de ambos.

El código metonímico de doble inferioridad de mujeres y traducción está en la base de multitud de otras metáforas. Esta doble inferioridad surge de la oposición entre el trabajo productivo/activo (realizado por hombres y autores) y el trabajo reproductivo/pasivo (realizado por mujeres y traductoras/ es). Según Chamberlain, el trabajo de reproducción, ya sea de seres humanos (realizado por mujeres) o de textos (realizado por traductores/as) está por lo general minusvalorado e incluso despreciado en las estructuras jerárquicas que definen nuestra cultura, pese a ser absolutamente vital. Se concibe así a mujeres y traducción como elementos periféricos respecto de un centro: la traducción es secundaria respecto a la escritura y el traductor/a respecto a la autoría, de igual modo que los feminismos son periféricos respecto al patriarcado y las mujeres respecto a los hombres. Como señaló Florio (1603), "because they are necessarily defective, all translations are reputed females" (apud Simon 1996: 1). El motivo de que se sexualicen estas metáforas está claro para Chamberlain (1992: 66): así es más fácil justificar el poder entre el texto original y la traducción, es decir, lo que se presenta como un problema estético es en realidad una cuestión de poder.

De lo dicho se desprende que desde los ET es necesaria una mirada (auto) crítica que examine otras posibles metáforas y discursos teóricos desde una 
perspectiva feminista. Esto, además, tendrá consecuencias positivas para la traducción, ya que subvertir el carácter negativo (reproductivo/pasivo) de las mujeres basándose en su estatus tradicionalmente devaluado cuestiona también la concepción secundaria y minusvalorada que se le otorga a la profesión de traducir. Pero además de esto, desde los ET se puede proponer una nueva retórica de la traducción que desconstruya las jerarquías entre los sexos y los textos, y que sustituya el lenguaje cliché por una terminología capaz de transmitir el juego activo de identidades que confluyen en la traducción. Esta nueva retórica, lejos de borrar la marca de género, podría mantenerlo estratégicamente para resignificarlo, liberándolo de su carga opresora patriarcal.

Un bosquejo sobre el que construir una nueva retórica de la traducción podría basarse en metáforas de la doble $e^{4}$ experiencia, ya que tanto la traducción como los feminismos reivindican (desde los márgenes en los que se encuentran) que su sujeto cuenta con el privilegio de tener acceso tanto a la realidad dominante como a la alternativa/deseable. Así, las y los feministas conocen tanto la estructura patriarcal en la que viven como el sistema de igualdad por el que abogan, del mismo modo que las y los traductores conocen tanto la lengua/cultura origen y la lengua/cultura meta.

Otra propuesta, de las muchas posibles, podría establecer una retórica basada en una diferencia no esencialista que parta de que tanto los feminismos como la traducción son herramientas importantes para el estudio y la comprensión crítica de la diferencia tal como ésta se (re)presenta en el lenguaje. En la traducción, se trata de la diferencia entre el texto reescrito y el original que otorga legitimidad para la creación y producción, en detrimento de la reproducción (retomando la ya mencionada idea de "translation as rewriting"). En cuanto a los feminismos, se trata de la diferencia entre los géneros, pero también entre cada persona individual respecto de cualquier otra (e incluso respecto de ella misma en diferentes momentos de su vida), evitando caer así en posturas esencialistas que mantienen que existe una esencia femenina y/o masculina que transciende los límites sociales y culturales. En lugar de ello, habría que sostener que la noción de identidad masculina y/o femenina responde (al menos en parte) a un constructo histórico, siendo el resultado de un proceso discursivo complejo.

4. Utilizo la noción de doble experiencia para destacar los dos extremos del abanico, pero tratando de evitar un binarismo dicotómico. Quizás para ello resultaría más apropiado hablar de múltiples experiencias, pues sus fronteras tan difusas crean una gama ilimitada de posibilidades entre ambos extremos. 
2.1.2. Nuevas concepciones de la traducción en paralelo a las propuestas de los feminismos

Los feminismos han salido y salen enriquecidos de su relación con los ET, pero lo que pretendo aquí es, a la inversa, mostrar cómo a los discursos teóricos de traducción puede resultarles de utilidad el hecho de observar, reflexionar y aplicar determinados conceptos centrales de los feminismos.

En este sentido, la autora Martín Ruano (2006: 28) sostiene que establecer paralelismos entre la evolución de la definición de 'mujer' y 'traducción', en tanto que objetos de estudio de ambos movimientos, resulta muy beneficioso para los ET. Así, igual que los feminismos actuales desesencializan y cuestionan el concepto biológico de 'mujer' como punto de partida estable para cualquier teoría y política feminista, se puede cuestionar también que la traducción surja únicamente a partir de un texto original material y con significación estable (desesencializando así la definición ideal y tradicional de traducción). De modo semejante, poner en evidencia que la definición de 'mujeres' se ha atribuido por heterodesignación (dado que como grupo histórico han carecido de poder para autoafirmarse) por y para beneficio del sistema patriarcal, invita a cuestionar que la definición de traducción haya corrido por cuenta ajena durante muchos años, identificándola con un conjunto de reglas idealizadas e inalcanzables que se debían cumplir (de ahí su carácter prescriptivo) para obtener la réplica perfecta. Pero incluso cuando dentro del paradigma descriptivo se autodefine la traducción, suele considerarse que es traducción aquello que la sociedad/cultura meta clasifica como tal, igual que es 'mujer' lo que una sociedad/cultura concibe como tal. De esta forma, igual que desde los feminismos se presenta como cuestionable que una mujer no tenga poder para ser lo que quiera ser (teniendo que comportarse como se espera de ella para lograr aceptación social), desde una perspectiva crítica los ET podrían cuestionar que la traducción tenga que comportarse como se espera de ella (para ser considerada como tal), pues eso impediría enfoques experimentales que difieran de lo hegemónico.

Por poner un segundo ejemplo de nuevas concepciones de la traducción a partir de conceptos centrales en los feminismos, se podría argumentar que igual que los feminismos se erigen como un discurso de resistencia contra los valores patriarcales y neoliberales que ejercen una opresión y discriminación de género, la traducción puede constituir, en propuesta de Venuti, "a cultural means of resistance against multinational capitalism and the political institutions to which the current global economy is allied" (2008: 18). Es más, dado que el capitalismo multinacional responde primordialmente a concepciones misóginas, de la interacción entre feminismos y traducción podría concebirse 
un medio de resistencia cultural más heterogéneo que reivindique una intervención política para cuestionar ambos discursos dominantes y fomentar una reflexión crítica. Así se pone de relevancia el activismo social implícito en ambas disciplinas, superando aquellas críticas que acusaban a los discursos teóricos (sobre traducción y feminismos) de permanecer en el terreno de la divagación filosófica estéril.

Estos son tan sólo dos ejemplos de las muchas aportaciones pendientes de explorar con las que los feminismos pueden contribuir a abrir debates los ET.

\subsection{Plano historiográfico}

El pasado otorga legitimidad. Si para cualquier disciplina es fundamental tener un pasado, para los ET adquiere una importancia estratégica, dada la "juventud" de la disciplina entendida como tal: sólo en época reciente las reflexiones teóricas sobre la traducción dejaron de verse como una rama de otras disciplinas (lingüística, literatura, filología, etc.), para consolidarse y establecerse como área independiente, aunque con una honda vocación transdisciplinar.

2.2.1. Reelaboración de la historia de la traducción teniendo en cuenta a las traductoras

La construcción de ese pasado implica examinar la historiografía de la traducción. Durante largos períodos históricos la escritura se consideró una actividad productiva/masculina, lo que impidió el acceso de muchas mujeres al mundo literario como autoras. La traducción, en su consideración de actividad reproductiva, sí se percibía como femenina y de esta forma se convirtió en válvula de escape con la que algunas mujeres pudieron entrar en el mundo literario. Con todo, en algunos casos, como en la Gran Bretaña del s. XVIII, se consideraba que sólo los hombres podían traducir desde las prestigiosas lenguas clásicas, quedando confinadas las traductoras a textos literarios efímeros y secundarios en idiomas modernos (cf. Agorni 2005).

Hubo autoras, sin embargo, que no pudieron reprimir sus ansias de escritura y produjeron sus propias obras. El férreo control por el poder autorial las llevó en ocasiones a firmar sus obras con pseudónimos de hombre (cf. Bartrina 2001 para examinar el caso de Caterina Albert, conocida como Victor Català), o bien a presentarse a ellas mismas como traductoras (Kord 1994: 12), como estrategia para conseguir ver sus libros publicados evitando la crítica social que deslegitimaría sus obras incluso antes de comenzar su lectura. De esta forma la traducción actúa como instrumento liberador, al rescatar a las 
mujeres de un silencio impuesto como autoras permitiéndoles la entrada en el mundo literario como traductoras, pero también como instrumento opresor, porque las condena al margen del discurso.

En cualquier caso, estas traductoras/autoras se hicieron visibles en prefacios, dedicatorias, notas a pie de página, correspondencias privadas, etc., donde reflexionaban sobre el acto de traducir y sobre las limitaciones que condicionaban su práctica. Sin embargo, estos metatextos se han perdido y silenciado, de forma que la concepción que la mitad de la humanidad tenía sobre el acto de traducir queda excluida de la historia de la traducción.

Después de que los feminismos hayan puesto en evidencia esta carencia, una actitud (auto)crítica de los ET debería conducir a la recuperación de estos materiales y metatextos que darán a conocer la intervención de esas mujeres en los movimientos culturales e intelectuales de su época y su forma de enfrentarse a la opresión patriarcal. Con esto se contribuirá a una definición más precisa y completa de la disciplina que, de otra forma, carece de una parte fundamental de su historia. En esta línea se sitúan los trabajos de Hannay (1985), Krontiris (1992), Robinson (1995), Delisle (2002) o Agorni (2005), que completan la historiografía de la traducción con las reflexiones teóricas de Aphra Behn, Suzanne du Vegerre, Germaine de Staël, las traductoras inglesas de la época Tudor, Susannah Dobson, Elizabeth Carter, Jane Wilde, Clémence Royer, Albertine Necker de Saussure, Julia E. Smith, etc. En nuestro contexto más inmediato, destaca la recuperación de la escritora y traductora gallega Emilia Pardo Bazán (cf. Freire 2006) o de las traductoras catalanas del s. XX (cf. Godayol 2007).

\subsubsection{Recuperación de autoras ignoradas}

A pesar de que las mujeres quedaban casi siempre excluidas de la autoría, a medida que los feminismos fueron democratizando la vida pública y privada algunas pudieron publicar sus obras. Un enfoque de género revela que ha sido un canon patriarcal el que ha definido tradicionalmente la estética y el valor literario, de forma que privilegia las obras de autores en detrimento de las autoras, sean éstas clásicas o actuales, próximas o de sistemas culturales distantes. En consecuencia, muchas obras de estas escritoras están perdidas, es decir, existen muchas autoras a las que no se ha traducido pese a escribir obras relevantes que, según los feminismos, sí se habrían traducido de haber sido elaboradas por un autor, como ilustran Ríos y Palacios (2006) en su análisis de la traducción al gallego de literatura irlandesa a comienzos del s. XX. 
Los ET tienen en este aspecto una labor primordial que desarrollar, interrogando qué se traduce, quién decide lo que se traduce, y en base a qué criterio se realizan estas elecciones como primer paso para poner fin a esta actitud discriminatoria. Desde la traducción se puede también contribuir a transformar el canon literario contemporáneo, optando abiertamente por una recuperación de los trabajos de estas autoras silenciadas, lo que a su vez enriquecería sumamente el campo de la traducción. En mi opinión, no se trata de colaborar con las mujeres por existir unos lazos de solidaridad universales, o por el hecho de que sean mujeres (lo cual constituiría una actitud paternalista), sino porque sus obras resultan relevantes, aunque esa relevancia permanezca oculta al no adecuarse a los criterios estipulados por el canon patriarcal.

En lo que respecta a la recuperación de las escritoras clásicas mediante su reescritura a otras lenguas, la traducción puede servir como un instrumento para contextualizarlas, incorporando comentarios en los que las traductoras/ es debatan sobre las razones que llevaron a esas obras a ser ignoradas. En efecto, "recovery and commentary" son dos de las estrategias sugeridas por Massardier-Kenney (1997: 59-62) en su propuesta, en la que también repasa algunos de los ejemplos más destacados de autoras recuperadas mediante su traducción comentada. A ellos se une la labor de Rayor (1991) cuando traduce y comenta a diecisiete poetas griegas de la antigüedad, de DendrinouKolias (1990) al traducir la autobiografía de Elisavet Moutzan-Martinengou (1989), de Kadish junto a la misma Massardier-Kenney (1994) con su edición desde el francés de Translating Slavery, o de multitud de otras traductoras que con sus reescrituras contribuyen a difundir a autoras silenciadas.

En este punto es necesario evaluar también el trabajo de resignificación que la traducción puede ofrecer a autoras feministas que, aunque 'canónicas' en su contexto originario, fuesen apropiadas por otros discursos. Un caso claro son las traducciones de Rosalía de Castro a inglés realizadas por Kathleen March, en las que la traductora recupera el mensaje feminista de la autora que había sido neutralizado en su contexto original por las jerarquías nacionalistas patriarcales para poder unirla más fácilmente a la 'causa común' (cf. González Liaño 2002).

En relación a las autoras poscoloniales, distantes o de culturas minoritarias, la traducción resulta un canal comunicativo esencial, y con frecuencia el único, que tienen las autoras para compartir sus subjetividades más allá de sus fronteras. Aún así, como sostiene Spivak (1993), se necesita una gran dosis de discernimiento para no caer en la actitud reprobable (por mucho que sea benévola) de traducir todo lo que venga de una cultura minoritaria, 
poscolonial o distante, para saber evaluar los riesgos éticos derivados de hablar por otras, y para evitar servir tan sólo a los intereses occidentales de los mercados de traducción, en lugar de entender la situación real en la cual se escriben e inscriben las textualidades originales. Además de las traducciones de la propia Spivak de la autora bengalí Mahasureta Devi, en este campo destaca el importante trabajo de Tharu y Lalita (1993) con la traducción de textos de autoras indias editados en dos volúmenes.

En última instancia, el hecho de que se traduzcan textos de autoría femenina entre diferentes lenguas y culturas pondrá en común experiencias de muy distintas mujeres contribuyendo a romper la presunción patriarcal de que el hombre es heterogéneo y la mujer homogénea y a constatar que el género no es un principio unificador para todas las mujeres, sino que configura la identidad junto a otras variables. Asimismo, si nos referimos específicamente a la traducción de obras feministas, desde los ET se podría analizar en qué medida la traducción ha contribuido a la expansión del movimiento feminista por todo el mundo a través de traducciones que pusieron en contacto diferentes metodologías que antes no se conocían.

\subsection{Plano crítico}

\subsubsection{Crítica de traducciones de obras feministas}

Pese a todas las dificultades de las autoras para acceder a la voz pública primero, y para que sus obras sean traducidas después, a medida que los feminismos avanzan se hace más fácil que algunas autoras publiquen obras que, siendo magistrales, se traduzcan posteriormente a otras lenguas. Un análisis de esos materiales muestra que en ocasiones se crean traducciones rigurosas y conscientes recurriendo a diferentes estrategias que los ET podrán evaluar para utilizar en otros contextos. Sin embargo, en muchas otras ocasiones el análisis crítico desvela la existencia de traducciones de libros de autoras (especialmente autoras feministas) realizados por "phallotranslators, inadequate interpreters of women's writing, given an observable reliance on engrained phallocentric assumptions" (Henitiuk 1999: 473). O bien desde la invisibilidad, o bien desde una visibilidad que aprovechan para presentarse como objetivos y fieles en lugar de dar a conocer su posición ante el texto, estos falotraductores desvirtúan el original, incorporándolo a la ideología dominante a través de una traducción patriarcal.

Uno de los casos más paradigmáticos es la traducción al inglés de Le deuxième sexe, de Simone de Beauvoir, realizada por el zoólogo Howard Parshley, a quien se le encargó la tarea por considerar que el libro trataba sobre 
sexualidad y reproducción. A partir de trabajos críticos sobre esta traducción (cf. Simons 2001; Moi 2004; Castro 2008) se puede comprobar cómo el traductor suprimió cerca del quince por cierto del original francés en el primer volumen, y en el segundo eliminó unas sesenta páginas, omitiendo hechos 'incómodos' (largas secciones sobre los logros de las mujeres en la historia, hazañas de mujeres que desafiaron los estereotipos de género, tabúes sobre relaciones lesbianas, descripciones del arduo trabajo de las amas de casa, etc). Entre las consecuencias tan serias de este comportamiento, destaca la definición de Beauvoir por parte de la audiencia anglófona como una filósofa inconexa e intelectualmente inmadura, y sobre todo el cruce de acusaciones entre las feministas francófonas y anglófonas por interpretar diferentes postulados a partir de un mismo texto que, en realidad, llegaba a sostener tesis dispares ${ }^{5}$.

También se produjeron tensiones a partir de las traducciones en inglés de los textos de Hélène Cixous, Luce Irigaray y Julia Kristeva debido a la "tendency to neglect full textual explanations of concepts and rhetorical strategies" (Simon 1996: 107), lo que atenuó la recepción y comprensión de las posturas del feminismo francés entre sus colegas angloamericanas.

Estas "falotraducciones" no sólo afectan a textos canónicos, sino también a cualquier otro texto (literario) en que la autora rompa con la pretensión patriarcal. Un caso es el estudiado por Miguélez-Carballeira (2005) sobre la traducción a inglés de Bruce Penman del libro de Esther Tusquets El amor es un juego solitario. En su texto en español Tusquets otorga importancia a la fisiología textual/sexual, mostrando el placer sexual femenino a través de imágenes textuales. A pesar de esto, en Love is a Solitary Game se detectan importantes omisiones y neutralizaciones semánticas en aquellos pasajes en los que la autora menciona el órgano sexual femenino. Así, "su sexo tibio, húmedo, pegajoso y fragante, su sexo flor en el pantano, su sexo nido, su sexo madriguera, en el que retroceden todos los miedos, este sexo que es para Ricardo un punto de partida..." se convierte en inglés en "there's something warm, moist, clinging and fragrant, like a flower in the marsh where she wandered so long, like a nest, like the lair of an animal, something which takes away all fear. For Ricardo it is a starting point". E incluso la descripción de una felación centrada en la óptica de la mujer como "mientras agita con cuidado entre dos dedos, estrecha luego en la palma de una mano firme y cálida, oprime entre sus pechos, resigue con los pezones erizados, se desliza en la boca" (no

5. Desde 1999, año del cincuentenario de la obra, las académicas feministas exigían a la editorial Random House una segunda traducción de la obra en inglés. Finalmente, en 2006 Jonathan Cape (con los derechos sólo para el ámbito británico) anunció una nueva traducción de Constance Borde y Sheila Malovany-Chevallier. 
mencionando siquiera el pene y otorgando al varón una posición pasiva y ausente), se convierte en inglés en "as she flipped his organ gently between two fingers, squeezed it with a firm, warm hand, pressed it between her breasts, stropped it against her erected nipples, and finally slid it into her mouth" (introduciendo una referencia explícita al órgano sexual masculino y un "finally" que sugiere que, desde la óptica del varón, éste llega a su objetivo).

A partir de estos ejemplos se demuestra la necesidad de una actitud (auto) crítica en los ET para desenmascarar falotraducciones con las herramientas que aportan los feminismos. Pero además, estas herramientas resultan también fundamentales para desvelar aquellos casos en los que la traducción desempeña un papel clave en la canonización de ciertos textos como feministas, aun no siendo considerados como tal en su contexto original.

\subsubsection{Crítica de paratraducciones de obras feministas}

Hay un espacio de análisis íntimamente ligado a la traducción que por lo general queda fuera del estudio de la crítica: la paratraducción (Garrido 2005). Este concepto, normalmente aplicado a la traducción literaria, se desarrolla a partir de elementos que envuelven y presentan el texto como títulos, prólogos, notas, anuncios, cubierta o aspectos gráficos, que Genette (1987) agrupó bajo el nombre de paratextos y que, al abordar la traducción de un libro, también hay que trasladar a la cultura de llegada. Así, provisionalmente, podría decirse que la paratraducción es la traducción de paratextos. La paratraducción no es un espacio reservado únicamente a traductores/as, sino que aparecen otros mediadores (correctores de pruebas, revisores lingüísticos, editores) que suelen tener más poder para decidir cómo se presenta la obra en la sociedad de llegada, actuando también conforme a una determinada ideología muy propensa a dejarse influir por criterios económicos. Entre todos estos paratextos, el nivel icónico tiene una relevancia fundamental, pues "provocará repercusións na propia textualidade e, consecuentemente, modificará a lectura que do texta meta fará o destinatario final" (Yuste 2001: 850). Así, cuando los paratraductores/as escogen un título o imagen de cubierta siguen una estrategia comunicativa ideológica que

determina unha recepción, unha lectura ideolóxica e mesmo apunta ao tipo de público ao que vai dirixido. Tamén pode espectaculizar por medio desas imaxes ofrecendo tamén dese xeito adscrición xenérica, contido e argumento do libro. (Garrido 2005: 36)

Así pues, aunque la paratraducción no sólo corresponde a quien traduce, sí influye poderosamente en la recepción de la traducción, y por ese motivo los ET deberían también prestar atención a la transmisión de ideología a nivel 


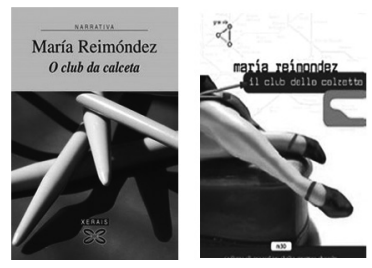

Figura 1
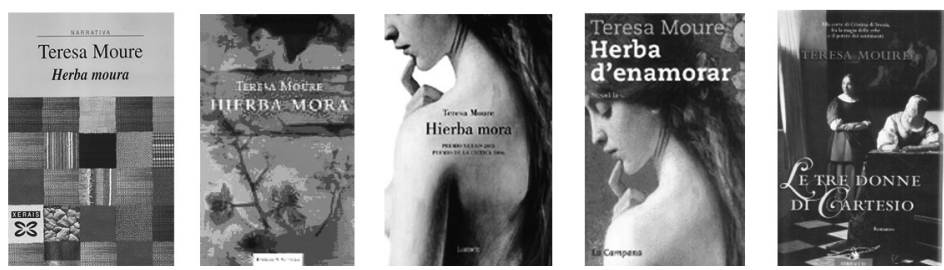

Figura 2

paratextual. De nuevo, una perspectiva feminista pone de manifiesto cómo de manera nada ingenua se alteran (quizás tergiversan) los paratextos feministas del original, sobre los que las autoras sí tuvieron más poder de decisión. Resulta fácil ilustrarlo analizando las cubiertas de dos obras superventas escritas por dos escritoras explícitamente feministas de la narrativa gallega contemporánea. En primer lugar (fig. 1), María Reimóndez y su $O$ club da calceta (2006), que en la edición original se presentaba con unas agujas de calcetar y fondo lila (color de connotación feminista). En la traducción al italiano, sin embargo, las agujas de calcetar quedan muy relegadas porque la imagen destaca las largas (y depiladas) piernas de una mujer tumbada en un sofá, sin que responda a ningún pasaje de la novela. El segundo ejemplo (fig. 2) es el premiado Herba Moura (2005) de Teresa Moure. Los elementos gráficos de la edición original (un tapiz de diversos tejidos y colores) se sustituyen en la primera edición española por una imagen difusa de un rostro femenino mezclado con hierba mora; y en la segunda edición, por una imagen de una mujer (medieval) desnuda de espaldas, en actitud muy sensual. Esa misma imagen, pero mostrando el rostro completo, fue la escogida para la edición que titula el libro con una connotación directa a las propiedades de la "herba moura". En la cubierta de la traducción italiana se decide destacar uno de los contextos temporales en los que se desarrolla la trama, con un título que coloca a las tres protagonistas en posición de dependencia del también personaje Descartes (Le tre donne di Cartesio). 


\subsection{Plano práctico: ¿hacia una tercera ola?}

Este recorrido por las interacciones entre feminismos y traducción incluye también el plano práctico, es decir, la aportación de los feminismos a la comprensión del comportamiento traductor en el proceso por el que un texto en una lengua se convierte en otro texto en otra lengua. A diferencia del plano crítico, no se trata tan sólo de analizar las soluciones que (ya) se han adoptado en el producto, sino de estudiar el proceso por el cual se adoptan unas u otras.

\subsubsection{Comportamiento traductor respecto a la representación textual ${ }^{6} \mathrm{de}$ mujeres y hombres}

Una de las áreas de estudio consiste en analizar el comportamiento traductor en este proceso teniendo en cuenta la representación textual/lingüística de mujeres y hombres en los textos, dada la estrecha relación entre las prácticas discursivas y sociales.

Las herramientas metodológicas con las que llevar a cabo este análisis las proporciona el marco de los ET caracterizado por el giro cultural o ideológico ya mencionado, así como la lectura que desde los feminismos se hacía de la ideología dominante: si no se suscribe de forma consciente una determinada ideología, se está traduciendo (inconscientemente) conforme a la dominante.

Desde el giro cultural traducimos entre culturas, pero no podemos olvidar que las fichas con la que jugamos la partida (de la traducción) son palabras, frases, textos, discursos. De ahí que, además de la perspectiva de los estudios culturales, para poder desenmascarar cómo los valores culturales e ideológicos subyacen a determinados discursos, sea de utilidad la lingüística crítica o el análisis crítico del discurso (cf. Fairclough 1995; Fowler et al. 1979). Pero se trata de examinar cómo la persona traductora lee primero y transmite después su lectura de la representación lingüística de hombres y mujeres en el texto, por lo que lo más apropiado es recurrir a la lingüística crítica feminista, que conjuga lingüística crítica con feminismo. Por ese motivo, su evolución está directamente relacionada con estas dos disciplinas. Y si durante años se concebía como una lingüística feminista de la segunda ola (la que tenían a su disposición y aplicaron las traductoras canadienses), ahora resulta más productivo que desde los ET se valoren las renovadas posibilidades de análisis que ofrece la "third wave feminist linguistics" que propone Mills (2003 y 2008).

6. Aunque defenderé un cambio del texto al discurso como unidad de análisis, utilizo la noción de textual por oposición a paratextual. 
Esta lingüística feminista de la tercera ola supone ubicar la unidad de análisis en el discurso, lo que la aproxima al "feminist critical discourse analysis" de Lazar (2005). Así, abandona las afirmaciones globales sobre usos sistemáticos del lenguaje para centrarse en el análisis puntual y específico de cada enunciado (pues existen diferentes posiciones lectoras en cada contexto); evita los análisis aislados que pueden dar lugar a generalizaciones universales sobre hombres y mujeres, para realizar análisis siempre contextualizados que permitan entender con rigor cómo se establecen los límites de significación; y rechaza considerar las categorías hombre y mujer en exclusiva, para concebirlas junto a otras variables (edad, raza, clase, etc.) con las que siempre interactúan.

Aplicar esto a la traducción crea un nuevo marco metodológico que podríamos denominar 'third wave feminist translation', y cuyo primer ámbito de análisis consistiría en abordar la representación discursiva de mujeres y hombres en el texto original, siempre de forma puntual, contextualizada y teniendo en cuenta la interacción del género con otras variables. Esta representación discursiva puede materializarse en diferentes niveles, como la palabra (a través de términos concretos o, en algunos idiomas, del género lingüístico) o la frase (frases hechas, refranes, etc. en los que no hay marcas de género pero implícitamente se refieren a hombres o mujeres), y siempre teniendo en cuenta que dejan de ser elementos aislados para convertirse en constituyentes de un discurso: es decir, teniendo en cuenta que una misma palabra o frase puede utilizarse en dos discursos de forma que represente a su referente de forma dispar, por lo que nunca se podrán leer de forma absoluta.

Una vez abordada esta representación discursiva, surge el segundo ámbito de análisis: considerar qué problemas de traducción plantea esa representación, teniendo en cuenta las (im)posibilidades de representación (lingüística y cultural) de estas y estos mismos referentes en la lengua meta, es decir, examinar los problemas de traducción entendidos en su dimensión discursiva, aunque puedan materializarse en palabras o frases. Por ejemplo, problemas de traducción ocasionados por palabras que, dependiendo del discurso, puedan tener como referente a mujeres y/o hombres ('children' como 'hijas', 'hijos', 'infancia'); problemas cuando estamos ante un masculino del que desconocemos si tiene valor genérico o específico, siendo eso relevante para la traducción ('tíos' como 'uncles', 'uncles and aunts', 'aunt and uncles', etc.); problemas cuando un texto que no explicita el sexo del/a referente sí debe hacerlo explícito en la lengua meta ('you're tired' como 'estás cansado' o 'cansada'); problemas cuando un texto sí menciona explícitamente el sexo del/a referente pero la lengua meta no necesita, aunque pueda, hacerlo explícito ('escritora' 
como 'writer' o 'women writer'); o, en general, problemas éticos que puedan surgir al traducir un discurso que los feminismos califiquen de reprobable. Pero además la tercera ola invita a los ET a considerar qué problemas no se plantean (pese a existir) por haber realizado una lectura inconsciente de la representación discursiva y que tendrán como consecuencia una reescritura acorde a la ideología dominante. No se trata únicamente del "Male-As-Norm Principle" (Braun 1997: 3) por el que, en caso de desconocer el sexo del referente, en la traducción se opta por el masculino a no ser que existan estereotipos en sentido opuesto, sino que se incurre en lo que se considerarían errores de traducción incluso desde una posición hegemónica. Son errores como los que se ilustran en estos ejemplos, extraídos de un ejercicio con alumnado de la Universidade de $\mathrm{Vigo}^{7}$, en el que 16 de 30 estudiantes tradujeron "one experiences one's pregnancy differently, every time" por "un vive o seu embarazo de xeito...", y 23 de 30 reescribieron "he's a very famous gynaecologist. His patients are very happy with him" como "é un xinecólogo moi famoso. Os seus pacientes están moi contentos...", creando situaciones inconcebibles con hombres embarazados y en consultas de ginecología. Cabe aquí un breve inciso para resaltar la necesidad de una (auto)crítica en las teorías pedagógicas de la traducción que conduzca, como reclama Susam-Sarajeva (2006), a explorar el papel de la docencia en generar actitudes críticas y conscientes sobre lo que implica traducir.

La imposibilidad posible de la traducción, por decirlo con Godayol (2000: 123), requiere buscar provisionalidades tácticas con las que resolver temporalmente esos problemas. En esta tercera fase, la más sugerente, es donde los feminismos plantean a los ET la necesidad de una actitud (auto)crítica para desarrollar nuevos debates a nivel práctico que planteen la traducción del género dentro del marco de la traducción feminista de la tercera ola. Uno de estos debates lo propone la traducción no sexista (cf. Castro 2006), que evalúa cómo afectan a la traducción las políticas de planificación lingüística de género. Así, a partir de una lectura consciente y no dominante de la representación discursiva de mujeres y hombres, la traducción no sexista propone estrategias de reescritura que consideren el contexto de la traducción (función del texto, público, tipo de representación lingüística, pares lingüísticos, limitaciones de cada tipología textual como poesía, traducción jurada, doblaje, etc.) pero que también valoren los intertextos de la lengua meta (el uso cada vez mayor de lenguaje no sexista en la lengua meta al escribir textos originales).

7. Trabajo empírico realizado con alumnado de la asignatura Tradución e cultura inglés/ galego (Lic. Traducción, Universidade de Vigo) en 2005/06. 
Considerando contextos e intertextos, las estrategias de traducción no sexista están determinadas por la contingencia discursiva, y por ello exigen una reflexión constante y tienen una validez únicamente provisional.

En cualquier caso, la tercera ola de la traducción feminista añade además dos nuevas dimensiones a la lectura y transmisión ideológica de la representación discursiva de mujeres y hombres. Primero, dado su interés en el contexto, invita a examinar no sólo los textos literarios (como hasta ahora se ha hecho casi exclusivamente, tanto desde la escuela canadiense como desde las posteriores propuestas), sino toda tipología textual: en este sentido, son interesantes los análisis de De Marco (2006) para la traducción audiovisual, y de Sánchez (2007) sobre la traducción del discurso científico. Pero, además de la traducción literaria, científica y audiovisual, en la actualidad vivimos en la era de las comunicaciones globales, lo que implica que cada vez estén más presentes en nuestras vidas otro tipo de traducciones. Esto abre un nuevo interrogante, ya que muchos de estos documentos tienen autoría desconocida, y otros ni siquiera se presentan como traducciones ante nuestros ojos. Habría pues que analizar también cómo estos dos condicionantes afectan (o no) a la lectura y reescritura discursiva del género.

Y segundo, dado su interés en el discurso (y en la palabra sólo como parte constituyente de un discurso que adquiere significación en su conjunto), habría que examinar además cómo la ideología afecta (o no) a la lectura y reescritura de los elementos discursivos que tienen como referentes a mujeres y hombres. Hasta ahora se ha teorizado y practicado traducción a partir de textos de ideología explícita y conscientemente feminista (escuela canadiense, Díaz-Diocaretz, etc.) o de ideología explícitamente misógina (Levine). Es momento de preguntarse cómo abordar la representación discursiva de mujeres y hombres cuando los textos no manifiestan abiertamente una ideología determinada. Esto conduce a nuevas preguntas: ¿Qué lecturas puede realizar la tercera ola de la traducción feminista para desenmascarar la ideología cuando ésta no es patente? ¿Si no es explícita, significa que es inconsciente (y por lo tanto, dominante)? ¿En base a qué debe constituirse la ética de la traducción?

\subsubsection{Comportamiento paratraductor respecto a la representación textual y paratextual de mujeres y hombres}

Generalmente se considera que las y los traductores son operadores principales del proceso de traducción, pero con frecuencia su capacidad de elección está supeditada a las decisiones funcionales que tomen otros agentes mediadores: los paratraductores/as (personas encargadas de la corrección de pruebas, revisión lingüística, edición, intermediación con cliente, cliente, 
mecenas, agencia de traducción, etc.), que suelen tener más poder para intervenir en el proceso. Estos paratraductores/as se encargan de revisar la traducción (adecuándola normalmente, pero no siempre, a su ideología consciente o inconsciente), aunque al final sea el traductor/a quien deba responsabilizarse públicamente de las elecciones de la traducción. De esta forma, un análisis en el marco de la tercera ola de la traducción feminista quedará manco a menos que interrogue también el papel de las y los paratraductores en dos aspectos. Primero, en recomendar o exigir una determinada lectura y reescritura traductora de los elementos discursivos que representan a mujeres y hombres. Segundo, en su propia interpretación y posible revisión de esos elementos tal y como aparecen en la reescritura traductora, cuando las ideologías de ambos/ as profesionales no es coincidente. Ambos puntos quedan ilustrados, en el contexto alemán y austriaco, por un interesante trabajo de Wolf (2006).

Además de estas relaciones de poder entre quien traduce y quien paratraduce elementos textuales (o discursivos), en la traducción literaria existen también una serie de espacios paratextuales que se deben traducir y/o crear para acompañar al texto. No cabe duda de lo beneficioso que resultaría para los ET abrirse a este nuevo campo de análisis para examinar el proceso por el cual las paratraductoras/es adoptan unas $u$ otras decisiones a la hora de trasladar aquellos elementos paratextuales (o paradiscursivos) que verbal o icónicamente representan a mujeres y hombres.

\section{Conclusiones}

A lo largo de este artículo he presentado diferentes áreas de análisis con las que potenciar el debate entre los ET y los feminismos desde una perspectiva (auto)crítica. Sólo esta perspectiva permitirá evaluar las interrelaciones pasadas y construir sobre ellas nuevos horizontes productivos. Por un lado, superando la (dominante) propuesta de la traducción feminista canadiense para nutrirse de nuevas aproximaciones prácticas al proceso de traducción y paratraducción enmarcadas dentro de la tercera ola de la traducción feminista. Por el otro, potenciando las interrelaciones también en un plano conceptual, crítico o historiográfico, entre otros muchos posibles. Tantos que por cuestión de espacio han quedado fuera campos de análisis no menos importantes, como podrían ser las diferencias entre mujeres y hombres respecto a la traducción desde una perspectiva neurobiológica y neurolingüística (una disciplina todavía muy joven y reciente sin resultados concluyentes); la influencia de una subjetividad masculina o femenina en la traducción (si el sexo es o no un factor que influya en la lectura y reescritura que hace la traductora o traductor); la influencia del sexo en el tipo de traducción (los tipos de textualidades 
que más traducen las mujeres y los hombres, si hubiere diferencias significativas); o las condiciones laborales en las que se desempeña la profesión desde un enfoque de género (porcentaje de traductoras y traductores que trabajan por cuenta ajena o que son autónomos en traducción literaria o no literaria, porcentaje de las y los que están licenciados o posgraduados en traducción, enfermedades laborales que afectan a traductoras y traductores, etc.). Como puede comprobarse, muchas son las líneas reflexivas que se pueden establecer sobre los estudios de traducción a partir de su interacción con los feminismos, lo que sin duda permitirá continuar potenciando interesantes debates en años venideros.

\section{Referencias bibliográficas}

AGORNI, Mirella. (2005) "A Marginal(ized) Perspective on Translation History: Women and Translation in the Eighteenth Century". Meta 50:3. pp. 817-830. AlthUSSER, Louis. (1975) "Ideología y Aparatos Ideológicos de Estado". [Trad. Albert Roises Qui]. Escritos. Barcelona: Laia. pp. 107-172.

ARrojo, Rosemary. (1994) "Fidelity and the Gendered Translation". TTR 7:2. pp. 147-163.

Arrojo, Rosemary. (1995) 'Feminist, 'Orgasmic' Theories of Translation and Their Contradictions". Tradterm 2. pp. 67-75.

Bartrina, Francesca. (2001) Caterina Albert/Víctor Català. La voluptuositat de l'escriptura. Vic: Eumo.

Bassnett-McGuire, Susan. ([1980]1991) Translation Studies. Londres \& Nueva York: Routledge.

BouRdieu, Pierre. (1998) La dominación masculina. [Trad. Joaquín Jordà]. Barcelona, Anagrama.

BRAUN, Friederike. (1997) "Making Men out of People: the MAN principle in translating genderless forms". En: Kotthoff, Helga \& Ruth Wodak (eds.) 1997. Communicating Gender in Context. Ámsterdam \& Filadelfia: Benjamins. pp. 3-30.

CASTRO VÁzQUEZ, Olga. (2006) Tradución e cambio social. Elementos de análise para unha tradución non sexista. Tesina de DEA inédita. Biblioteca de la Facultad de Traducción. Universidade de Vigo.

Castro VÁzquez, Olga. (2008) "(Para)Translated Ideologies in Simone de Beauvoir's Le deuxième sexe: The (Para)Translator's Role". En: Seruya, Teresa \& Maria Lin Moniz (eds.) 2008. Translation and Censorship in Different Times and Landscapes. Newcastle: Cambridge Scholars Publishing. pp. 130-146.

Chamberlain, Lori ([1988]1992) "Gender and the Metaphorics of Translation". En: Venuti, Lawrence (ed.) 1992. Rethinking Translation. Discourse, Subjectivity, Ideology. Londres \& Nueva York: Routledge. pp. 57-74. 
Cronin, Michael. (2000) "Ideology and Translation". En: Classe, Olive (ed.) 2000. Encyclopedia of Literary Translation into English. Londres: Fitzroy Dearborn. pp. 694-696.

De MARCo, Marcella. (2006) "Audiovisual Translation from a Gender Perspective". The Journal of Specialised Translation 6. pp. 167-184.

DelisLe, Jean Delisle (dir.) (2002) Portraits de traductrices. Ottawa: Université d'Ottawa y Artois Presses Université.

DendidRou-Kolias, Helen. (1990) "Empowering the Minor: Translating Women's Autobiography". Journal of Modern Greek Studies 8. pp. 213-221.

Díaz-DiocaretZ, Miriam. (1985) Translating Poetic Discourse: Questions on Feminist Strategies in Adrienne Rich. Ámsterdam y Filadelfia: Benjamins.

D’HULST, Lieven. (1992) "Sur le rôle des métaphores en traductologie contemporaine". Target 4:1. pp. 33-51.

FAIRClough, Norman. (1995) Critical Discourse Analysis: the critical study of language. Londres: Longman.

Freire López, Ana María. (2006) "Emilia Pardo Bazán, traductora: una visión de conjunto". En: Lafarga, Francisco \& Luis Pegenaute (eds.) 2006. Traducción y traductores, del Romanticismo al Realismo. Berna: Peter Lang. pp. 143-157.

FOWLER, Roger; Bob Hodge; Gunther Kress \& Tony Trew. (1979) Language and Control. Londres: Kegan Paul.

Garrido Vilariño, Xoán Manuel. (2005) "Texto e paratexto. Tradución e Paratradución”. Viceversa 9-10. pp. 31-39.

GenetTE, Gérard. (1987) Seuils. París: Seuil.

GODARD, Barbara. (1990) "Theorizing Feminist Discourse/Translation". En: Bassnett, Susan \& André Lefevere (eds.) 1990. Translation, History and Culture. Londres y Nueva York: Pinter. pp. 87-96.

GODAYOL, Pilar. (1998) "Interviewing Carol Maier: a woman in translation". Quaderns. pp. 155-162.

GODAYOL, Pilar. (2000) Espais de frontera: gènere i traducció. Vic: Eumo.

GODAYOL, Pilar. (2007) "Dona i traducció: del plaer a l'ofici". Quaderns Divulgatius, 32. (Associació d'Escriptors en Llengua Catalana). pp. 41-53.

GonzÁlez Liaño, Iria. (2002) "Tradución e xénero: o feminismo de Rosalía de Castro traducido ó inglés". Viceversa 7/8. pp. 109-130.

HANnAY, Margaret Patterson (ed.) (1985) Silent But for the Word: Tudor Women as Patrons, Translators and Writers of Religious Works. Kent, Kent State University Press.

Henitiuk, Valerie (1999). "Translating Woman: Reading the Female through the Male”. Meta 44: 3. pp. 469-484.

HeRMANS, Theo (ed.) (1985) The Manipulation of Literature. Essay in Translation Studies. Londres: Croom Helm. 
KADISH, Doris y Françoise Massardier-Kenney. (eds.) (1994) Translating Slavery: Gender and Race in French Women's Writing, 1783-1823. Kent: Kent State University Press.

KORD, Susanne. (1994) "The Innocent Translator: Translation as pseudonymous behaviour in eighteenth-century German women's writing". The Jerome Quarterly 9. pp. 11-13.

KRONTIRIS, Tina. (1992) Oppositional Voices: Women as Writers and Translators of Literature in the English Renaissance. Londres \& Nueva York: Routledge.

LAZAR, Michelle. (2005) Feminist Critical Discourse Analysis. Studies in Gender, Power and Ideology. Basingstoke: Palgrave.

LEFEVERE, André \& Susan Basnett. (1990) "Introduction: Proust's Grandmother and the Thousand and One Nights. The Culture Turn in Translation Studies". En: Bassnett, Susan \& André Lefevere (eds.) 1990. Translation, History and Culture. Londres \& Nueva York: Pinter. pp. 1-13.

LEVINE, Suzanne Jill. (1983) "Translation as (Sub)version: On Translating Infante's Interno". Sub-stance 42. pp.85-93.

LOTBINIÈRE-HARWOOD, Susanne de. (1991) Re-Belle et Infidèle. La traduction comme pratique de reécriture au féminin-The body bilingual. Translation as a rewriting in the feminine. Toronto: Women's Press.

MARTín RuAnO, María Rosario. (2006) "Gender(ing) Theory: Rethinking the Targets of Translation Studies in Parallel with Recent Developments in Feminism". En: Santaemilia, José (ed.) 2006. Gender, Sex and Translation. The Manipulation of Identities. Manchester: St. Jerome. pp. 27-37.

MASSARDIER-KENNEY, Françoise. (1997) "Towards a Redefinition of Feminist Translation Practice”. The Translator 3:1. pp. 55-69.

MiguÉlez-CARballeira, Helena. (2005) "Gender-related Issues in the English Translations of Esther Tusquets and Rosa Montero: Discrepancies between Critical and Translational Figurations". New Voices in Translaton Studies 1. pp. 43-55.

Mills, Sara. (2003) "Third Wave Linguistic Feminism and the Analysis of Sexism". Discourse Analysis Online 2:1. Versión electrónica: http://extra.shu. ac.uk/daol/articles/open/2003/001/mills2003001-paper.html

Mills, Sara. (2008) Language and Sexism. Cambridge: Cambridge University Press.

MoI, Toril. (2004) "While We Wait: Notes on the English Translation of The Second Sex". En: Grosholz, Emily (ed.) 2004. The Legacy of Simone de Beauvoir. Oxford: Oxford University Press. pp. 37-68.

Moutzan-Martinengou, Elisavet. (1989) My Story. [Trad. Helen DendrinouKolias] Georgia: University of Georgia Press. 
Nouss, Alexis. (2000) "Structuralism, Post-structuralism and Literary Translation". En: Classe, Olive (ed.) Encyclopedia of Literary Translation into English. Londres: Fitzroy Dearborn. pp. 1351-1355.

RAYOR, Diane. (1991) Sappho's Lyre: Archaic Lyric and Women Poets of Ancient Greece. LA: University of California Press.

Ríos, Carmen \& Manuela Palacios. (2006) "Translation, Nationalism and Gender Bias". En: Santaemilia, José (ed.) 2006. Gender, Sex and Translation. The Manipulation of Identities. Manchester: St. Jerome. pp. 71-79.

RoBinson, Douglas. (1995) "Theorizing Translation in a Woman's Voice: Subverting the Rhetoric of Patronage, Courtly Love and Morality". The Translator 1:2. pp.153-175.

SÁNCHEZ, Dolores. (2007) "The Truth about Sexual Difference. Scientific Discourse and Cultural Transfer". The Translator 13:2. pp. 171-194.

SIMON, Sherry. (1996) Gender in Translation. Londres \& Nueva York: Routledge.

Simons, Margaret A. (2001) Beauvoir and The Second Sex. Feminism, Race and the Origins of Existencialism. Maryland: Rowman \& Littlefield.

SNELL-HoRnBy, Mary. (1990) "Linguistic Transcoding or Cultural Transfer? A Critique of Translation Theory in Germany". En: Bassnett, Susan \& André Lefevere (eds.) Translation, History and Culture. Londres \& Nueva York: Pinter. pp. 79-86.

SPIVAK, Gayatri Chakravorty. (1993) Outside in the Teaching Machine. Londres \& Nueva York: Routledge.

STEINER, George. ([1975)1992) After Babel: aspects of language and translation. Nueva York: Oxford University Press.

Susam-SARAJEVA, Sebnem. (2006) "A Course on 'Gender and Translation' As An Indicator of Certain Gaps in the Research on the Topic". En: Santaemilia, José (ed.) 2006. Gender, Sex and Translation. The Manipulation of Identities. Manchester: St. Jerome. pp. 161-176

Tharu, Susie y Ke Lalita. (1993) Women Writing in India. 600 B.C. to the Present. Nueva York: The City University of NewYork.

VEnUtI, Lawrence. (2008) "Translation, Simulacra, Resistance". Translation Studies 1:1. pp. 18-33.

Vidal Claramonte, Carmen África. (1998) El futuro de la traducción: últimas teorías, nuevas aplicaciones. Valencia: Alfons Magnànim.

von Flotow, Luise. (1991) "Feminist Translation: Contexts, Practices and Theories". TTR 4:2. pp. 69-84.

VON Flotow, Luise. (1997) Translation and Gender. Translation in the Era of Feminism. Manchester: St. Jerome.

Wolf, Michaela. (2006) "The Female State of Art: women in the 'translation field"'. En: Pym, Anthony; Miriam Shlesinger \& Zuzana Jettmarová (eds.) 
2006. Sociocultural Aspects of Translating and Interpreting. Ámsterdam y Filadelfia: Benjamins. pp. 129-141.

YUSTE FRÍAS, José. (2001) "Traducir en la red: textos nuevos para nuevas traducciones". En: Barr, Anne; Rosario Martín Ruano \& Jesús Torres (eds.) 2001. Últimas corrientes teóricas en los estudios de traducción y sus aplicaciones. Salamanca: Universidad Salamanca. pp. 848-857. 